\title{
360 Degrees of Security: Can VR Increase the Sustainability of ISA Trainings?
}

\author{
Tobias Fertig, David Henkelmann, and Andreas E. Schütz \\ Faculty of Computer Science and Business Information Systems \\ University of Applied Sciences Würzburg-Schweinfurt \\ Sanderheinrichsleitenweg 20, 97074 Würzburg, Germany \\ \{tobias.fertig, andreas.schuetz\}@fhws.de, david.henkelmann@student.fhws.de
}

\begin{abstract}
What companies need are employees who have an appropriate level of information security awareness (ISA). This paper examined ways to increase existing ISA knowledge. The core of the work was to investigate the possibility of a more sustainable effect of knowledge enhancement in relation to ISA through virtual reality $(V R)$. For this purpose, VR training and traditional video training were compared within a subject study. In order to create the most efficient video training possible, a qualitative literature research was first conducted on the topic of knowledge transfer in general. This was followed by the development of didactic guiding principles for an optimized learning video. Both training courses were then tested. Theoretically, a sustainable effect of increasing ISA knowledge through $V R$ training has been proven. However, within the scope of the subject study, no sustainable increase in ISA knowledge can be proven through VR training in comparison to video training. Therefore, the didactic and immersive possibilities of VR technology need to be further explored in follow-up studies.
\end{abstract}

\section{Introduction}

Numerous surveys and studies have shown that most data breaches are caused by oversights or mistakes made by employees [3]. Often there is no malicious intent behind such mistakes. The employees are simply not educated and sensitized enough. For companies, employees with a low information security awareness (ISA) level are currently one of the greatest risks for their own organization [20]. A study from 2019 dealing with incidents of data theft or espionage in German companies, confirms this argument [3]. Of all the companies surveyed, $24 \%$ stated that they had been the victim of social engineering at least once between 2017 and 2019 [3]. According to this statistics, social engineering attacks are the second most common attack method used by hackers [3]. This development shows that no matter how strong the number of technological defense barriers is, employees who work with these technologies remain vulnerable. Therefore, technology alone does not protect against information security and privacy risks. Employees must be integrated into the information security process in order to reduce their vulnerability and that of their own organization [20].

Properly trained employees are able to adapt to the changing risks faster than technological and procedural countermeasures [16]. To do so, they need to be integrated into the organization's information security concepts [20]. Therefore, implementing and improving security awareness programs and ISA trainings is important for organizations to ensure that risks are mitigated. However, cyber security threats that exploit human behavior are becoming more sophisticated, so ISA training must not only address new attack methods, but also opportunities that improve the quality of ISA training [4] [18].

This is where the technology of virtual reality (VR) comes into play. VR offers a completely new possibility of imparting knowledge. For the first time, VR technology has made it possible to create a virtual space that completely blanks out reality for the user.

Since more and more experts are developing new concepts for sensitizing employees for ISA it is not surprising that a very recent paper published by Ulsamer et al. [22] deals in detail with the topic of training ISA through VR. The authors compare two groups of subjects: one group learned ISA compliant behavior through a VR learning unit, while the control group only completed a text and slide based learning unit. At the end, the learning success of the two groups was measured and compared. The findings of their study were that the subjects learned more effectively via VR. Therefore, the question arises: Are VR trainings a more efficient way to increase the ISA of employees? In order to answering this question, the work of Ulsamer et al. [22] will be continued.

In the following, we begin by defining our six research questions. We then summarize related work. In doing so, we address four different topics: ISA, social engineering, virtual reality as well as cognitivism. The next section, "Methodology", describes our overall approach to the work. In the section "Thematic structure 
of the video", the ISA topics presented in the two learning units are described in more detail. This is followed by an explanation of the study procedure in the section "Conducting the study". In the related subsections, the collected results from the study are presented. These collected and presented results will then be discussed in the section "Discussion" and our research questions will also be answered. Finally, we give a brief summary of the work and conclude with an outlook and future work.

\section{Research Questions}

Conducting a study on whether VR-based ISA training enables a sustainable sensitization for ISA of end users is the goal of this research. Therefore, a traditional video learning unit was created according to didactic multimedia guidelines. The approaches used are state of the art for multimedia and will be used within the video learning unit. The video content was optimized in regard to knowledge transfer in the topic of social engineering. The content was predefined since we reused the VR training video which already existed at our university. Afterwards we conducted a study which investigated the following research questions and for each of those we will have two hypotheses to consider in Section 6.3:

RQ1a. Does the use of video trainings increase the ISA of employees?

RQ1b. Is the knowledge acquired through video training retrievable over a longer period of time?

RQ2a. Does the use of VR training increase the ISA of employees?

RQ2b. Is the knowledge acquired through VR training retrievable over a longer period of time?

RQ3a. Does VR training increase the knowledge about social engineering as opposed to video training?

RQ3b. Does training through VR have a more sustainable learning outcome than training through a traditional video?

\section{Related Work}

\subsection{Information Security Awareness}

The term ISA can be understood as a person's individual degree of sensitization for a certain topic of information security [20]. ISA influences people's behavior, when they are working with information technology. This behavior can contribute positively to information security if people work in compliance with the IS-related guidelines and best practices of a company. The awareness of employees can be increased with awareness measures. However, in order to implement targeted ISA measures in the company, it must be understood how ISA is composed [20].

According to Schütz [20] ISA is a cognitive and affective construct i.e., a construct that includes cognitive aspects, such as logical and rational thinking, but also affective aspects, such as feeling, perceiving and acting instinctively. Schütz [20] defines the four aspects knowledge/ability, behavioral intention, salience and habit. These four aspects and the external factor of environmental constraints result in the degree of information security-compliant behavior that is reflected in the actions of employees. The aspect salience defines the attention given to a behavior. In addition, the aspect habit describes a behavior that is performed so frequently and internalized that it occurs automatically in certain situations in the future [23].

\subsection{Social Engineering}

In the process of this work, two awareness measures, both targeting the knowledge aspect, were compared. This comparison serves to gain insights into how ISA can be influenced via the knowledge aspect in relation to the different media, VR and animated video. The learning units should educate about social engineering in general, show characteristics of a phishing email and specifically inform about the main topic of pretexting.

Social engineering describes the targeted manipulation of one person by another [15]. The person is to be influenced in favor of the social engineer without him or her noticing and is to be led to an action desired by him or her. The well-being and self-interest of the victim plays no role and is ignored [15].

Pretexting is also a social engineering method. Pretexting is defined as creating an invented scenario to get a chosen victim to reveal information or perform an action [11]. Pretexting goes beyond making up a lie. In some cases, an entirely new identity is created, which is then used to manipulate the receipt of information. Social engineers pretend to be people who hold certain jobs and roles that they themselves have never held [11]. A pretexting attack consists of four phases [11]: Obtaining information, building trust, exploiting trust, and carry out the actual project. Obtaining knowledge is used to find out everything there is to know about the victim. Afterwards, the social engineers use this information to build trust with the victim. This is followed by a seemingly harmless request to which the 
victim is supposed to respond. Finally, the social engineer has the opportunity to carry out his goal.

As mentioned at the beginning, the two awareness measures that were compared in the context of this paper are designed to increase the knowledge aspect.

\subsection{Virtual Reality - A New Way to Learn}

A VR learning environment is a closed, threedimensional computer-based learning environment [5] The representation of this environment takes place by means of so-called VR glasses. These present the threedimensional computer-generated environment and hide the real world [5]. Therefore, VR technology offers learners a virtual classroom in which they can act proactively and become part of the learning experience themselves. Learners are placed in a situation in which they are confronted with a problem and have to learn how to deal with this problem interactively. The resulting situational handling of the learning object offers the possibility to individually design and change the VR environment and to influence it through one's own actions. Learning from the first-person perspective promotes direct authenticity and immediate learning experience. Furthermore, tactile and auditory stimuli of VR technology create just-in-time feedback that allows learners to reflect on their own actions within the VR environment and thus induce learning success [5].

One of the biggest advantages of VR is the way it is presented. VR enables explorative representation through image-analogue modelling of the environment. This means that VR objects are presented in their real size, scope as well as detail, which makes more complex representations possible. As an example, it would be conceivable to illustrate complex biological processes in the body for learners in three dimensions in virtual space. The ability to recreate any real situation virtually has yet another advantage. In VR, situations can be simulated that are too expensive or dangerous in reality [5].

Two important terms that are often mentioned in connection with VR are immersion and presence. Immersion describes the extent to which a technology is able to present a holistic living illusion of reality [19]. Presence, on the other hand, describes a psychological state of consciousness. This state is the subjective feeling of being present in the virtual world, even though one is still physically present in the real environment [19]. In the literature, Wirth et al. [25] define presence as a two-dimensional construct. This construct is composed of the sense of self-location in the immersive environment and the recognised opportunities for interaction with the environment. Presence is said to have a positive influence on the transfer of learning from the virtual environment to reality. However, Busch et al. [6] found no significant correlation between presence and learning transfer in their study. Nevertheless, the study shows that the userfriendliness of the virtual application was rated higher by the test persons as presence [6].

The comparison of VR-based training with traditional education shows certain advantages in terms of theoretical knowledge transfer, but especially in the practical transfer of skills. Abstract theoretical problems can be presented in virtual three-dimensional space to support theoretical thinking. Furthermore, practical training also benefits from the new technology. By providing immersive learning experiences, a wide variety of processes can be simulated realistically in a cost-effective and safe manner [5].

\subsection{Cognitivism}

There are different learning theories that describe how skills or knowledge are represented in the brain. In the history of learning research, there are three major directions, so-called paradigms, which provide theories on learning [7]. A distinction is made between behaviorism, cognitivism and constructivism [17].

When creating the animated learning unit, cognitivist approaches were used. This is because cognitivism attempts to create a basis for explaining learning processes and offers approaches for improving the process of creating learning units.

Cognitivism is concerned with how thinking works. It attempts to make statements about human memory and its ability to store and retrieve knowledge, as well as to process information [10]. Cognitivist learning theories often include the analogy of human information processing and computer information processing. Thus, short-term memory is considered comparable to the working memory of computers, while long-term memory has parallels to a hard drive [11]. Cognitivism understands all processing of experiences and sensory impressions that result from interaction with the environment as processes of learning. These sensory impressions and experiences must be filtered, ordered and processed [17]. The model of human information processing from Gagné and Driscoll [9] describes the processes that take place in memory.

According to the model of Gagné [9], information is processed in the brain in a multi-stage process. After this process, people themselves become impulse generators for the environment. Be it by carrying out what they have learned or simply answering a question.

The reception of sensory stimuli occurs via the environment. The brain receives impulses via the individual sense organs such as the eyes, ears, sense of smell or taste. These are perceived by the sensory registration. However, sensory registration holds 
received information for only a very short time. Information is either forgotten at this point or selected information is transferred to short-term memory. In the short-term memory, a comparison is now made with information from the long-term memory. The shortterm memory also has the ability to relate different information from the long-term memory to the newly acquired impressions [17].

The aim of cognitivist learning theories is to optimize the design of learning processes [10] [11], [17]. Digital media offerings based on cognitivist approaches are usually multimedia and learningadaptive learning content. Examples of this would be learning videos that use visual and auditory elements to convey targeted knowledge [17].

\section{Methodology}

To answer the research questions, four thematic steps were defined. The first step was a literature review on the basics of learning didactics. Since ISA trainings should be compared, the topic of knowledge transfer had to be analyzed. Therefore, literature describing the learning process in memory was searched for learning theory assumptions. Because cognitivist learning theories aim to optimize the design of learning processes, they were also used within the research. Based on this, multimedia guidelines were derived, because the learning unit should be an animated video. The elements cognitive load, text, image and sound were considered in detail and guidelines for a targeted didactic usage were uncovered from the literature. Finally, the guidelines for a combination of the individual elements text, image, and sound were evaluated. These derived multimedia guidelines served as the basis for the creation of the video in order to convey the knowledge as effectively as possible.

In the second step, the animated learning video was created. For this purpose, the pre-existing VR videos were analyzed and the knowledge to be covered was extracted. The participants should be taught four core topics through the learning unit: What is social engineering in general, how to recognize a phishing email, what is pretexting and how does it work, as well as how to protect oneself against pretexting. These four topics were structured and prepared. Subsequently, the content concept of this video was created with the help of the prepared topics. The final creation of the animated video was then realized with the tool Vyond [24]. In Vyond, we combined the media and multimedia guidelines as well as the content concept to create an animated learning unit.

The third step was to design the study and create the necessary components to conduct the study. In order to find out whether the use of VR training sustainably increases the ISA of end users, a quantitative field study was conducted. For this purpose, 24 test persons were divided into two groups. Each of the groups received a one-time presentation of one of the learning units. Knowledge was measured before the presentation, immediately afterwards and then at intervals of two, four and eight weeks. The chosen time period was intended to provide information about the retention performance of the test persons and to enable a comparison of the existing ISA knowledge after different time intervals. The knowledge test was carried out via surveys created in Limesurvey [14]. There was a total of five Limesurvey surveys for each group. The content of the surveys was based on the ISA topics presented in the learning units.

In the last step, the results of the surveys were checked by means of a distribution test. Within each group, the results of the first and second, the second and third, the third and fourth, as well as the fourth and fifth tests were compared using the paired t-test [8]. In this way, insights into the retention performance within each group could be gained. The two groups were also compared against each other. The goal was to determine whether the group that trained via the VR learning unit performed better than the group training with a conventional learning video. The results of the second to fifth tests of the groups were contrasted and again compared using the unpaired t-test [8]. The statistical tests within each group as well as across groups were designed to check for any significant abnormalities. These results, obtained from the statistical tests, formed the basis for the final discussion. Last but not least, in the discussion, the research questions were discussed, taking into account all the findings from the four steps, in order to give a final answer.

\section{Thematic Structure of The Video}

Since we reused pre-existing VR videos, the contents taught had to be identical to those of the VR videos provided. At the beginning, the main topic of the video was mentioned i.e., hacking. Afterwards, the term hacker was briefly explained and then the topic transitioned to social engineering. Now there was a detailed explanation of social engineering and one of the methods of social engineering: Phishing. Therefore, the participants were shown the most important characteristics of a phishing email and tips on how to protect themselves using an example phishing email. This concluded the first part of the video. The main part of the video dealt with pretexting. Moreover, the difference to phishing was explained. Then, a presentation of the four stages of pretexting (cf Section 3.2) followed. The individual contents of the four stages were explained to the test persons and presented using a 
subsequent scenario. The video shows how a hacker can steal data from a company by using the method of pretexting. During the whole scenario, what the attacker does and in which of the four stages they are, was explained. Afterwards, possible countermeasures that can protect against an attack were explained. In the last part of the video, all topics were repeated once again and the recommendations for protection against phishing and pretexting were listed.

\section{Conducting the Study}

The study was conducted with 24 subjects who were divided into two groups. Group one, which will be called the non-VR group, was shown the video created as a traditional learning unit. The other group, called the VR group, was given an environment in a virtual world that represented the same content. The presentation of the learning units took place once for each participant. The test persons were tested a total of five times. The five tests for the test persons had to be completed by the participants immediately before viewing the learning units, immediately afterwards and then at intervals of two, four and eight weeks after viewing. This period was chosen in order to be able to test the retention performance. Moreover, whether the different learning units also have different effects on the retention period of the recallable knowledge was determined. We have to mention, that our institution does not have an Institutional Review Board. Therefore, we could not receive a favorable ethics opinion.

The non-VR group received an explanation of the study procedure at the beginning. The test persons were informed about the questioning intervals and the onetime viewing of the video. Some participants were trained remotely, as presence was not necessary for the non-VR group. Participants who could not be physically present, received the explanation of the procedure via video chat. After the introductory explanation, the test persons were informed about the structure of the test questionnaire and could clearify any questions. Afterwards, the first test to determine the current level of knowledge of the test persons and general sociodemographic data was carried out. The participants were informed in the introduction that it was not held against them if they were unable to answer any questions during the first session. We wanted to enable a direct comparison of the knowledge level before and after viewing the learning video in order to be able to determine the knowledge acquisition. If the test was successfully completed, the learning video was presented and the person tested again. This was the end of the first part and the participants were invited for a new test in a fortnight. Therefore, after two, four and eight weeks, the participants were provided with the survey links with the request to complete the tests.

The VR group also received an introductory explanation of the general procedure of the study. A special requirement was that the subjects of the VR group had to be physically present for the first part of the study. Afterwards, we explained the structure of the test questionnaire, as we did with the non-VR group. Again, it was noted that not answering the test questions in the first run is not held against the participants, as it serves to determine the level of knowledge before viewing the learning unit. The respondents were also given the opportunity to ask questions before starting the questionnaire. After completing the first questionnaire, the test persons sat down on a chair and were familiarized with the use of the HTC Vive Pro VR glasses. The HTC Vive Pro was chosen because of its precise 360-degree control, headset tracking, realistic graphics, directional audio and HD haptic feedback for realistic movement and action in the virtual world. Likewise, the HTC Vive Pro was already available at the institution. After the test persons had familiarized themselves with the VR glasses, they were presented with the VR learning unit. At the end of the learning unit, the subjects were given the second questionnaire. In addition to the test questions, this contained questions about the VR experience. Like the non-VR group, the participants were invited to the follow-up tests in two, four and eight weeks.

\subsection{Preparation of the Questionnaires}

The questionnaire for the animated learning unit was structured as follows. The first part contains sociodemographic questions on age, current employment situation and data on how often a computer or mobile phone is used. In addition, four items ask general questions about information security. Two of the items ask whether the respondents are confronted with information security issues in their private or professional lives and how well they know about attacks against information security. Each respondent must answer these two items. The other two items, which relate to information security within the company or within the institution, are only answered by those who stated either "in an employment relationship" or "fulltime training at an educational institution" in the question about their current employment situation. This first part is only the subject of processing in the first survey. It is no longer included in the following surveys. The introductory questions are followed by the actual test questions which are shown in Table 1. 
Table 1 Point distribution ISA questions

\begin{tabular}{|l|l|}
\hline Question & Points \\
\hline What do you call the form of hacking in which the point of attack is the human being? & $1 \mathrm{P}$ \\
\hline Name the four stages of pretexting. & $4 \mathrm{P}$ \\
\hline Which is one of the best-known social engineering methods? & $1 \mathrm{P}$ \\
\hline Name three characteristics by which you can recognize a phishing mail. & $3 \mathrm{P}$ \\
\hline Which of the following sources of information does the hacker mostly use in step one of pretexting? & $1,5 \mathrm{P}$ \\
\hline In social engineering, the hacker tries to... (Name two) & $1,5 \mathrm{P}$ \\
\hline How do you protect yourself against pretexting? & $3 \mathrm{P}$ \\
\hline Which statements do not apply to pretexting? & $2 \mathrm{P}$ \\
\hline Imagine you were a hacker: What information about the victim would you look for in step one? & $3 \mathrm{P}$ \\
\hline Total Points & $\mathbf{2 0 ~ P}$ \\
\hline
\end{tabular}

All five questionnaires contain the same ISA test questions. Since the animated learning video is thematically identical to the VR videos given, the test questions were created based on it. The test questions consist of nine items and were combined into a second group of questions. As seen in Table 1, they deal with what has been learned from the video. Two items ask general questions about information security awareness in relation to social engineering. One item deals with the characteristics of a phishing email and the remaining items with the main topic of the learning unit, pretexting.

\subsection{Study Population and Sampling}

The subjects were not only randomly selected, but care was also taken to select subjects from different institutions to ensure that the study was as unbiased as possible. Test persons were selected from universities as well as from the free economy. These were distributed evenly in order to have two groups that were balanced. It should also be noted that there was no compensation for participation, neither monetary nor in any other form. The age of the subjects in the non-VR group $(\mathrm{N}=$ 12) ranged from 18 to 65 years. There were no participants in the range 36 to 50 years. Of the twelve participants in the non-VR group, seven (58.33\%) were studying or in full-time further education and four $(33.33 \%)$ were in employment. One $(8.33 \%)$ of the participants was not in any of the aforementioned. The average non-VR group participant was a student between 18 and 25 years old. They used the computer several hours a day and their smartphone daily. The average participant dealt with information security issues in everyday life and was rather familiar with information security attacks. Information security has a very high priority at the educational institution where they were enrolled. The educational institution was also very keen to actively promote information security.
The age of the participants in the VR group $(\mathrm{N}=$ 12) ranged from 18 to 65 years. There were no participants in the range of 26 to 50 years. Of the twelve participants, six $(50 \%)$ were studying or in full-time further education and five $(41.67 \%)$ were in employment. One $(8.33 \%)$ of the participants was not in any of the aforementioned. The average VR group participant was a student between 18 and 25 years old. They used the computer three to five times a week and their smartphone daily. The average participant did not deal with information security issues in everyday life and did not know anything about attacks on information security. Information security has a rather high priority at the educational institution where they were enrolled. The educational institution also tended to actively promote information security.

\subsection{Research Results and Analysis}

Since the Kolmogorov-Smirnov tests [8] indicated that the data were normally distributed, parametric tests were applied. To test whether the subjects' test scores differed significantly before and after participation in the learning unit, the paired t-test was applied within each group [8]. The paired t-test was also used to compare follow-up tests with their preceding tests in order to measure retention over the eight-week period [8]. The unpaired t-test enabled a comparison of the two scores of the non-VR and VR groups [8]. All tests performed were two-sided tests with a significance level of $p \leq 0.05$. Each result yielded:

- The significance level $\mathrm{p}$

- The $t$-value: $t$ can be negative as well as positive. Negative means that mean one is smaller than mean two. Positive means the opposite result.

- The h-value: $\mathrm{h}$ indicates whether the null hypothesis of equal medians can be rejected. Here $\mathrm{h}=1$ indicates that the null hypothesis of equal medians can be rejected and $h=0$ that it cannot be rejected. 
In the first run, the participants of the non-VR group achieved an average of 7.458 points $(\sigma=4.191)$ and in the second run, the participants achieved an average of 16.25 points $(\sigma=2.073)$. A significant difference in the score within the non-VR group in the first and second run of the ISA test could be demonstrated using a paired t-test with $\mathrm{p} \leq 0.05$ (see Table 1). The participants achieved a lower score in the first run, as the $\mathrm{t}$-value shows (see Table 1).

Table 2 Results T-Test First and Second Run non-VR Group

\begin{tabular}{|l|c|}
\hline Group & \multicolumn{2}{|c|}{ Paired T-Test } \\
\hline Non-VR Group & $\mathrm{p}=0,00002 \quad \mathrm{~h}=1 \mathrm{t}=-6,991$ \\
\hline
\end{tabular}

In the third run, the average score obtained was $16.08(\sigma=1.621)$, in the fourth run $15.792(\sigma=2.017)$ and in the last test the average score was $15.5(\sigma=$ 1.784).

On average, the subjects did not score significantly lower in the second and third runs. This was shown by the paired t-test with $p \geq 0.05$ (see Table 2). The t-value shows a slight decrease of the median in the third test.

Table 3 Results T-Test Second and Third Run non-VR Group

\begin{tabular}{|l|ll|}
\hline Group & \multicolumn{2}{|c|}{ Paired T-Test } \\
\hline Non-VR Group & $\mathrm{p}=0,825 \quad \mathrm{~h}=0 \quad \mathrm{t}=0,226$ \\
\hline
\end{tabular}

The subjects' performance also did not change significantly from the third to the fourth test. Due to the significance level, shown in Table $3, p \geq 0.05$, the null hypothesis of equal medians had to be assumed. The $\mathrm{t}$ value confirms a slight decrease in the median in the fourth test.

Table 4 Results T-Test Third and Fourth Run non-VR Group

\begin{tabular}{|l|ll|}
\hline Group & \multicolumn{3}{|c|}{ Paired T-Test } \\
\hline Non-VR Group & $\mathrm{p}=0,478 \quad \mathrm{~h}=0 \quad \mathrm{t}=0,736$ \\
\hline
\end{tabular}

The subjects' performance did not change significantly in the last test. This is shown by the paired $\mathrm{t}$-test with $\mathrm{p} \geq 0.05$. The $\mathrm{t}$-value again confirms a nonsignificant decrease in the median. Both values can be seen in Table 4.

Table 5 Results T-Test Fourth and Fifth Run non-VR Group

\begin{tabular}{|c|c|}
\hline Group & Paired T-Test \\
\hline Non-VR Group & $p=0,576$ \\
\hline
\end{tabular}

In the first test, the participants of the VR group scored an average of 3.625 points $(\sigma=1.860)$. The second run resulted in an average score of 15.29 points $(\sigma=1.912)$. The paired t-test performed, shown in Table 5 , showed $p \leq 0.05$. Thus, the null hypothesis of equal medians had to be rejected. The participants scored lower in the first round, as the t-value shows (see Table $5)$.

Table 6 Results T-Test First and Second Run VR Group

\begin{tabular}{|l|l|}
\hline Group & \multicolumn{2}{|c|}{ Paired T-Test } \\
\hline VR-Group & $\mathrm{p}=1,13 \mathrm{e}-9 \quad \mathrm{~h}=1 \quad \mathrm{t}=-18,648$ \\
\hline
\end{tabular}

Participants scored an average of 15.375 points $(\sigma$ $=2.123)$ in the third round, $15.75(\sigma=1.588)$ points in the fourth and $13.833(\sigma=1.863)$ points in the last round. Based on the mean values from test one to four, a steady increase in points could be seen. In the last test, the subjects' performance fell below the value of the second test.

The second and third runs did not score significantly lower. Based on the average score achieved in both test results and the t-value in Table 6, it can be seen that a slightly higher score was achieved in the third test. Table 6 shows the significance level $p$ $\geq 0.05$. Thus, the null hypothesis of equal medians was to be accepted.

Table 7 Results T-Test Second and Third Run VR Group

\begin{tabular}{|l|lc|}
\hline Group & \multicolumn{3}{|c|}{ Paired T-Test } \\
\hline VR-Group & $\mathrm{p}=0,897 \quad \mathrm{~h}=0 \quad \mathrm{t}=-0,133$ \\
\hline
\end{tabular}

The same applies to round three and four. Here, too, there was no significant difference in the scores. As the t-value shows (see Table 7), a higher average score was achieved in test four than in run three. The paired t-test performed, shown in Table 7, resulted in $p \geq 0.05$. This meant that the null hypothesis of equal medians was accepted.

Table 8 Results T-Test Third and Fourth Run VR Group

\begin{tabular}{|l|ll|}
\hline Group & \multicolumn{3}{|c|}{ Paired T-Test } \\
\hline VR-Group & $\mathrm{p}=0,319 \quad \mathrm{~h}=0 \quad \mathrm{t}=-1,043$ \\
\hline
\end{tabular}

In the last run, there was a significant difference compared to the fourth run. A decrease in the median can be seen. The paired t-test with $p \geq 0.05$ confirms the significance. Based on the t-value, it can also be seen that a higher average score was achieved in test four. The significance level and the t-value are listed in Table 8.

Table 9 Results T-Test Fourth and Fifth Run VR Group
\begin{tabular}{|l|ll|}
\hline Group & \multicolumn{3}{|c|}{ Paired T-Test } \\
\hline VR-Group & $\mathrm{p}=0,008 \quad \mathrm{~h}=1 \quad \mathrm{t}=3,232$ \\
\hline
\end{tabular}

Comparing the score differences from test two of the non-VR and VR groups, the unpaired t-test conducted showed that there is no significant difference between the two groups as $p \geq 0.05$. The non-VR group even scored slightly higher on average, as shown by the t-value in Table 9 and the mean scores of the two groups above.

Table 10 Comparison of Results non-VR Group and VR Group
Second Test
\begin{tabular}{|l|lll|}
\hline Group & \multicolumn{3}{|c|}{ Unpaired T-Test } \\
\hline Non-VR and VR difference & $\mathrm{p}=0,252$ & $\mathrm{~h}=0$ & $\mathrm{t}=1,178$ \\
\hline
\end{tabular}

The score differences between the two groups in test three are also not significant. The non-VR group again performed better, which is confirmed by the tvalue in Table 10. However, it can be seen that both mean values are converging. The null hypothesis of 
equal medians was accepted by the paired t-test with $\mathrm{p}$ $\geq 0.05$.

Table 11 Comparison of Results non-VR Group vs VR Group Third
Test
\begin{tabular}{|l|lcc|}
\hline Varable & \multicolumn{3}{|c|}{ Unpaired T-Test } \\
\hline Non-VR and VR difference & $\mathrm{p}=0,369 \quad \mathrm{~h}=0$ & $\mathrm{t}=0,919$ \\
\hline
\end{tabular}

Test four showed that both groups now have almost identical medians. The null hypothesis of equal medians was accepted, as the unpaired t-test again showed $\mathrm{p} \geq$ 0.05 (see Table 11). The t-value also confirmed the fact that the medians of the two groups are almost identical. The non-VR group only achieved a minimally higher average score.

Table 12 Comparison of Results non-VR Group vs VR Group Fourth

\begin{tabular}{|l|lll|}
\hline Variable & \multicolumn{3}{c|}{ Unpaired T-Test } \\
\hline Non-VR and VR difference & $\mathrm{p}=0,956$ & $\mathrm{~h}=0$ & $\mathrm{t}=0,0562$ \\
\hline
\end{tabular}

The final tests of both groups show in comparison that the VR group achieved a lower result, and the difference is statistically significant. The unpaired t-test showed $\mathrm{p} \leq 0.05$, which is why the null hypothesis of equal medians was rejected. The t-value also confirms the fact that the non-VR group achieved a higher result, and the difference is statistically significant (but with a small effect size). Both values are listed in Table 12.

Table 13 Comparison of Results non-VR Group vs VR Group Fifth

\begin{tabular}{|l|lll|}
\hline Variable & \multicolumn{3}{c|}{ Unpaired T-Test } \\
\hline Non-VR and VR difference & $\mathrm{p}=0,036$ & $\mathrm{~h}=1$ & $\mathrm{t}=2,239$ \\
\hline
\end{tabular}

In conclusion, all tests conducted, and results obtained indicate that the null hypothesis must be accepted. This means that subjects who were trained by a traditional video scored the same or higher in the ISA test than subjects who completed the VR training.

\section{Discussion}

Research question RQ1a can be answered in the affirmative. This is because the test persons achieved a significantly higher result in the second test. The second test was conducted after the video training in order to measure the increase in knowledge. Therefore, we assume that the video learning unit had a significant effect on the learning success of the test persons. However, the video learning unit was based on the didactic guidelines developed during the research process, which were compiled from scientific recommendations, and were used specifically for knowledge transfer. When creating a media learning unit, the learning success is strongly dependent on the targeted use of the didactic elements [13] [17]. In order to ensure the most effective learning possible, it is therefore recommended that future video trainings in the field of ISA take into account the didactic approaches as well as future scientific findings in the creation process. The answer to RQ1b is also yes. The test persons were able to maintain their performance continuously over the duration of five tests. There was no significant drop in the achieved score from test to test. Based on this fact, it can be assumed that the end users can recall the learned ISA knowledge over a longer period of time.

Research question RQ2a is answered in the affirmative. As with the video training, the subjects of the VR training also achieved a significantly higher score in the second test. Therefore, we assume that the VR technology has an influence on the transfer of knowledge. With regard to the pre-existing VR videos, it should be noted that didactic improvements still need to be made in some places that could increase the effectiveness of the VR training. One point of criticism of the VR training is the sound. At some points, the spoken word cannot be understood due to external influences such as wind noises. This resulted in information loss to the end user. Therefore, we recommend following the same procedure as for the creation of the animated video training. In order to improve the quality of the current VR training, all media components of the VR technology and the associated didactic concepts should be addressed. If such an optimization takes place, the potential can be significantly increased and thus also the quality of the ISA knowledge to be imparted. A first approach for a more effective transfer of learning from virtual to reality is offered by Rau et al. [19] with their design principles for VR. Their findings should be evaluated and tested for effectiveness in follow-up studies.

Research question RQ2b can also be answered with yes. The VR group achieved a slightly better result even after the VR training up to the fourth test. Thus, there was a continuous increase in recallable knowledge up to the fifth test. In answering research question RQ3b, this phenomenon will be examined again in more detail. In the last test, there was a drop in performance. The average score was lower than after the second test. Since the ISA knowledge was not refreshed, we expected that the performance would drop at a certain point. In practice, the accumulated ISA knowledge should be regularly refreshed in order to maintain a level of ISA that is useful for the organization. In the study conducted, it was shown that the first significant drop in performance occurred between the fourth and fifth test. This means that four weeks after the review of the learning unit, a decline in recallable knowledge began. Therefore, a first refresher of the ISA topics should take place after four weeks.

To answer research questions RQ3a and RQ3b, the findings from Section 6 were used. Research question 
RQ3a cannot be answered clearly considering the results from the statistical tests, because the VR group was able to record an average increase in points of 11.665 points from the first to the second test. In comparison, the nonVR group only achieved an average score increase of 8.792 points. Therefore, it is assumed that the VR training leads to a higher increase in knowledge in the area of social engineering in contrast to traditional video training. What also supports conducting further research regarding to RQ3a is the fact that after the second test, the average score achieved by the VR group was not significantly lower than that of the non-VR group. This was observed even though there was a difference in prior knowledge of ISA topics between the two groups, as the average respondent in each group was very different. As mentioned in Section 6.1, the regular participant of the non-VR group had a rather good knowledge of information security attacks and also dealt with information security issues in everyday life. In contrast, the regular participant in the VR group had no knowledge at all of attacks on information security and also had nothing to do with information security issues in everyday life. Therefore, the result of the second test indicates that the VR training almost completely compensated for the lower level of prior knowledge and led to an almost identical level of knowledge in the two groups.

For the research question RQ3b, we have to discuss several factors. Purely according to the statistical results, RQ3b has to be answered in the negative, because the non-VR group performed slightly better than the VR group in each of the five tests. Even though it did not perform significantly better until the fourth test. What is striking, however, is the fact that the VR group achieved a continuously improved test score from the second up to the fourth test. This phenomenon was also observed by Ulsamer et al. [22] in their work. Ulsamer et al. [22] were also able to demonstrate that the VR group achieved a slightly better result in the follow-up test. The findings from this work and those of Ulsamer et al. [22] speak for a sustainable transfer of knowledge through VR training. However, this study has not yet been able to prove a generally more sustainable learning success for VR training compared to conventional video training, which is why RQ3b must be answered in the negative. However, it should definitely be investigated where the increase in performance over time comes from, because this development is at odds with the way the visuospatial sketchpad of working memory works [1] [2]. The storage of the visuo-spatial noteboard is sketchy and information is only temporarily stored until it is overwritten by new visual information. One conjecture to explain this contradictory effect is the combination of "doing it yourself" with visual and auditory elements.
This means that not only the two subsystems, phonological loop and visuospatial sketchpad are addressed, as in traditional video training, but that a more realistic learning scenario is also presented. This more realistic learning scenario is made possible by VR technology, which can simulate a realistic environment [5]. Therefore, more in-depth research should be conducted in the area of immersion to find out whether the VR factor causes a subsequent increase in performance.

\section{Conclusion}

In summary, it can be said that VR training definitely causes a sustainable increase of ISA of end users. However, in comparison to an optimized video training, no advantage in terms of sustainability of knowledge acquisition could be proven during this research. This may be due to the fact that VR training is not yet fully developed, and the didactic potential is not fully used. Therefore, in follow-up studies, an investigation of the didactic possibilities, including the design principles of VR-technology established by Rau et al. [19], should be carried out in a new study. Only in this way we can show whether optimized VR training conveys ISA knowledge more sustainably than traditional training. For traditional video training, this work presents sustainable guidelines that improve the quality of knowledge transfer enormously. Also, looking at the processes of how knowledge is processed in the brain allows for a better alignment of the media used (image, sound and text) with regard to the Cognitive Load Theory [21] and the two subsystems of working memory (phonological loop and visuospatial sketchpad).

In the future, the following questions should be asked to enable a more comprehensive assessment of VR technology for ISA training, in practice:

- Why does ISA knowledge grow over a period?

- What happens when VR training is didactically optimized?

In addition, in future work, the timing of refreshing the imparted knowledge should be investigated. From when and how regularly must the topics be presented again so that a useful level of ISA is maintained in an organization? In the study conducted, it was shown that the first significant drop in performance occurred four weeks after the learning unit was reviewed. In the discussion, it was recommended to repeat the ISA topics again four weeks after the VR training. But what about advancing time? Does the time interval until knowledge decay increase with regular and continuous refreshing, because after a certain number of repetitions a transport of the ISA knowledge into the long-term memory takes place? In order to clarify these questions, further work 
should specifically investigate how often knowledge has to be presented and refreshed until it has passed into long-term memory.

Another point that should be added in the future is the implementation of a cost-benefit analysis. This enables a holistic view of VR training in practice. Moreover, it has to be verified if other ISA topics can also benefit from VR trainings. In conclusion, it can be said that VR technology will offer real added value for companies in the coming years. With further work in this area, widespread acceptance and implementation in practice are coming closer.

\section{References}

[1] Baddeley, A., 2000. The Episodic Buffer: a New Component of Working Memory? Trends Cogn. Sci. 4, 417-423. 6613(00)01538-2

[2] Baddeley, A., 1992. Working memory. Science 255, 556-559. https://doi.org/10.1126/science.1736359

[3] Berg, A., Niemeier, M., 2019. Wirtschaftsschutz in der digitalen Welt. Statista, Berlin. https://de.statista.com/statistik/daten/studie/164300/um frage/arten-von- computerkriminalitaet-inunternehmen-in-deutschland/

[4] BSI, 2012. LeitfadenInformationssicherheitITGrundschutz kompakt.

[5] Buehler, K., Kohne, A., 2019. Lernen mit Virtual Reality: Chancen und Möglichkeiten der digitalen Ausund Fortbildung, in: Groß, M., Müller-Wiegand, M.,

[6] Busch, M., Lorenz, M., Tscheligi, M., Hochleitner, C. Schulz, T., 2014. Being There for Real: Presence in Real and Virtual Environments and its Relation to Usability, in: Proceedings of the 8th Nordic Conference on Human-Computer Interaction: Fun, Fast, Foundational. Presented at the NordiCHI '14: The 8th Nordic Conference on Human-Computer Interaction, ACM, Helsinki Finland, pp. 117-126. https://doi.org/10.1145/2639189.2639224

[7] Cooper, P.A., 1993: "Paradigm shifts in Designing Instruction: From Behaviorism to Cognitivism", Educational Technology, 33(5), pp. 12-19.

[8] Field, A.P., 2009. Discovering Statistics using SPSS: and Sex, Drugs and Rock "n" Roll, 3rd ed. ed. SAGE Publications, Los Angeles.

[9] Gagné, R.M., Driscoll, M.P., 1988. Essentials of Learning for Instruction, 2nd ed. ed. Prentice Hall, Englewood Cliffs, N.J.

[10] Glaser, M., Weigand, S., Schwan, S., 2009. Mediendidaktik, in: Henninger, M., Mandl, H. (Eds.), Handbuch Medien- Und Bildungsmanagement: Qualitätsmanagement, Erwachsenenbildung, Mediengestaltung, Kommunikation, Personalentwicklung, Beltz-Pädagogik. Beltz, Weinheim, pp. 190-205.

[11] Gräsel, C., Gniewosz, B., 2015. Überblick LehrLernforschung, in: Reinders, H., Ditton, H., Gräsel, C., Gniewosz, B. (Eds.), Empirische Bildungsforschung.
VS Verlag für Sozialwissenschaften, Wiesbaden, pp. 19-24.

[12] Hadnagy, C., 2011. Social engineering: the art of human hacking. Wiley, Indianapolis, IN.

[13] Issing, L.J. (Ed.), 1987. Medienpädagogik im Informationszeitalter. Presented at the Symposium, Dt. Studien Verl, Weinheim.

[14] LimeSurveyLimeSurvey GmbH, 2021. URL https://www.limesurvey.org/de/impressum (accessed 15.03.2020).

[15] Mitnick, K.D., Simon, W.L., 2002. The art of deception: controlling the human element of security. Wiley, Indianapolis, Ind.

[16] Pattabiraman, A., Gupta, M., Swaminathan, K., Srinivasan, S., 2018. Fortifying Corporate Human Wall: A Literature Review of Security Awareness and Training., in: Gupta, M., Sharman, R., Walp, J. (Eds.), Information Technology Risk Management and Compliance in Modern Organizations, Premier Reference Source. Business Science Reference, Hershey, PA, pp. 142-175.

[17] Petko, D., 2014. Einführung in die Mediendidaktik: Lehren und Lernen mit digitalen Medien, 2nd ed, Bildungswissen Lehramt. Beltz, Weinheim.

[18] Prantner, K., 2009. Awareness und Lernen, in: Helisch, M., Beyer, M., Reibenspies, W. (Eds.), Security Awareness: neue Wege zur erfolgreichen MitarbeiterSensibilisierung, Praxis. Vieweg + Teubner, Wiesbaden, pp. 29-55.

[19] Rau, V., Niemöller, D., Berkemeier, L., 2019. Ist weniger mehr? - Designprinzipien für Virtual Reality Training aus kognitionspsychologischer Sicht. HMD Prax. Wirtsch. 56, 809-822. https://doi.org/10.1365/s40702-019-00546-1

[20] Schütz, Andreas. (2018). Information Security Awareness: It's Time to Change Minds! Presented at the International Conference on Applied Informatics ICDD Sibiu, Romania.

[21] Sweller, J., Ayres, P., Kalyuga, S., 2011. Cognitive Load Theory, 1. ed. ed, Explorations in the Learning Sciences, Instructional Systems and Performance Technologies. Springer, New York, NY.

[22] Ulsamer, P., Schütz, A., Fertig, T., Keller, L., 2021. Immersive Storytelling for Information Security Awareness Training in Virtual Reality. Presented at the Hawaii International Conference on System Sciences. https://doi.org/10.24251/HICSS.2021.861

[23] Verplanken, B. (Ed.), 2018. The Psychology of Habit: Theory, Mechanisms, Change, and Contexts. Springer International Publishing, Cham. https://doi.org/10.1007/978-3-319-97529-0

[24] VYONDTM, 2021. URL https://www.vyond.com (accessed 15.03.2020)

[25] Wirth, W., Hartmann, T., Böcking, S., Vorderer, P., Klimmt, C., Schramm, H., Saari, T., Laarni, J., Ravaja, N., Gouveia, F.R., Biocca, F., Sacau, A., Jäncke, L., Baumgartner, T., Jäncke, P., 2007. A Process Model of the Formation of Spatial Presence Experiences. Media Psychol. 9, 493-525. https://doi.org/10.1080/15213260701283079 